Available online on 15.10.2019 at http://ajprd.com
(c) 2013-19, publisher and licensee AJPRD, This is an Open Access article which permits unrestricted non-
commercial use, provided the original work is properly cited

Open 0 Access

Review Article

\title{
An Overview on Cystic Fibrosis
}

\section{Singireddy Swetha Reddy*, Varagandhi Susmitha, Jagiri Aishwarya Goud, Kadarla Rohith Kumar}

Department of Pharmacy Practice, Sree Chaitanya institute of Pharmaceutical Sciences Thimmapur, Karimnagar, Telangana, India

\begin{abstract}
A B S T R A C T
Cystic fibrosis is an autosomal recessive disease. It is caused by mutations in the CFTR (cystic fibrosis transmembrane conductance regulator) gene on chromosome 7 that codes for a protein transmembrane conductance regulator (CFTR) protein which functions as a transmembrane cAMP-activated chloride channel. CFTR also affects other ion channels, most notably blocking the influx of sodium into the cell through the epithelial sodium channel. The CFTR abnormality has been shown to produce a number of changes in the airway, including acidification and decreased water and ion transit. A pulmonary exacerbation of CF is usually identified by an increase in cough and sputum and a decrease in pulmonary function. Disease manifests in many organs, but most notably the upper and lower airways, pancreas, bowel, and reproductive tracts. Pulmonary function testing is a major tool for evaluating and monitoring disease state and progression in CF. Spirometry is the commonly used pulmonary function test. Management of CF requires good nutrition and appropriate supplementation of vitamins and pancreatic enzymes. The Cystic Fibrosis Foundation recommends the following treatments as having a high certainty of substantial net benefit, grade A, for moderate-to-severe disease: inhaled tobramycin, dornasealfa, ivacaftor, and inhaled aztreonam. Preventing or treating intestinal blockages - oral rehydration and osmotic laxatives (incomplete blockage) and hyperosmolar contrast enemas (complete DIOS). Antibiotics are the major components of CF treatment and are administered chronically (e.g. inhaled antibiotics, macrolides used for their immunomodulatory properties) or intermittently to prevent, eradicate, control or treat respiratory infections. Lumacaftor (200 mg) + ivacaftor (125 mg), Orkambi, is the first approved CFTR corrector and potentiator combination therapy.
\end{abstract}

Keywords: Cystic fibrosis (CF), Cystic fibrosis transmembrane conductance regulator protein (CFTR), Pulmonary function.

A R T I C L E I N F O: Received 06 May 2019; Review Completed 18 July 2019; Accepted 10 Oct 2019; Available online 15 Oct 2019
Cite this article as:
$\begin{aligned} & \text { Singireddy SR, Varagandhi S, Jagiri A G, Kadarla RK, An Overview On Cystic Fibrosis. Asian Journal of Pharmaceutical } \\ & \text { Research and Development. 2019; 7(5):80-91.DOI: http://dx.doi.org/10.22270/ajprd.v7i5.535 }\end{aligned}$
*Address for Correspondence:
$\begin{aligned} & \text { Dr. Rohith Kumar Kadarla, Asst Professor, Department of Pharmacy Practice, Sree Chaitanya Institute of Pharmaceutical } \\ & \text { Sciences,Thimmapur, Karimnagar, Telangana, India }\end{aligned}$

\section{INTRODUCTION:}

$\mathrm{I}$ n 1949, Lowe et al. postulated that cystic fibrosis must be caused by a genetic defect from the autosomal recessive pattern of inheritance of the disease. High levels of salt in the sweat of patients with cystic fibrosis suggested an abnormality in electrolyte transport from the sweat gland. Quinton postulated that sweat ducts in these patients were impermeable to chloride. Further studies led to the hypothesis that the faulty chloride channel must be situated in the apical membranes of the lung surface or glandular epithelium to explain the respiratory and systemic organ failure associated with cystic fibrosis. Researchers now know that cystic fibrosis is an autosomal recessive disorder of exocrine gland function most commonly affecting persons of Northern European descent at a rate of $1: 3500$. It is a chronic disease that frequently leads to chronic sinopulmonary infections and pancreatic insufficiency. The most common cause of death is end-stage lung disease ${ }^{1-4}$.

Cystic fibrosis is an autosomal recessive disease, caused by mutations of a gene on chromosome 7 which encodes for a chloride channel in the membranes of epithelial cells. 
Reduced chloride conductance results in viscid secretions and organ damage in the respiratory and reproductive tracts and the pancreatic and hepatobiliary ducts. Life expectancy is reduced and the main cause of death is respiratory failure due to progressive bronchiectasis and persistent lung infections with virulent organisms such as Pseudomonasaeruginosa, Staphylococcus aureus and Burkholderiacepacia. Other complications of cystic fibrosis include pancreatic insufficiency, diabetes, osteoporosis, liver disease and gallstones. Patients are treated in specialist centres with a complex treatment regimen that includes chest physiotherapy, nutritional supplements, pancreatic enzymes,mucolytics (e.g. nebulised DNase), longterm oral and nebulised antibiotics (e.g. colistin, tobramycin) and frequent courses of intravenous antibiotics (e.g.ceftazidime, meropenem, tobramycin).

There are approximately 8000 people living with cystic fibrosis in the UK and 1 in 25 of the population is a carrier of the cystic fibrosis gene mutation.1 The outlook has improved dramatically because of improved diagnosis and management: median survival in the UK is now 31 years. It is estimated that the life expectancy for a child born with cystic fibrosis in 2000 will be _50 years. Survival into adulthood brings with it issues of sexuality, fertility and potential pregnancy. This paper provides an overview of the current issues surrounding cystic fibrosis and pregnancy ${ }^{44}$.

In the 1980s, surveys showed that most people with cystic fibrosis were not married or sexually active. Recent studieshave shown, however, that sexual adaptation is normal and that sexual activity is not delayed when compared with adolescents without cystic fibrosis. Menstruation generally occurs slightly later in girls with cystic fibrosis and bodyweight is the most significant determinant of menarche. Once menstruation is established, the majority have regular cycles, with amenorrhoea most likely to occur in girls with poor lung function. Nearly all men with cystic fibrosis are infertile but women with cystic fibrosis have relatively fertility, despite having thickened cervical mucus which does not show the usual cyclical variation. An Aberdeen study6 highlighted that $72 \%$ of women with cystic fibrosis thought that having children was important but that $26 \%$ had never discussed fertility issues with a health professional. Other studies have shown that women and girls with cystic fibrosis are not well informed about reproductive issues.

Contraception is important for those wishing to avoid pregnancy and this needs to be discussed early, as unplanned pregnancy can be disastrous. Choice of contraceptive method can be difficult because of potential interactions with cystic fibrosis and its treatment but the risks of contraception need to be balanced against the risk of unplanned pregnancy. The combined oral contraceptive pill (COC) is the most frequently used method. There are concerns that it could be less effective in women with cystic fibrosis, in view of the frequent courses of antibiotics required; however, there have been no pregnancies reported as a result of COC failure. It is important to advise on the use of additional contraception (for example, condoms) when a woman is taking antibiotics. Long-term methods, such as Depo-Provera ${ }^{\circledR}$ (Pfizer Ltd, Walton-on-the Hill, Surrey), Implanon ${ }^{\circledR}$ (Organon, Cambridge, Cambs) and the Mirena ${ }^{\circledR}$ (Bayer plc, Newbury, Berks) intrauterine system, are highly effective and avoid concerns regarding malabsorption. Longterm use of Depo-Provera can, however, aggravate osteoporosis, a common complication of cystic fibrosis. If termination of pregnancy is required, the usual methods (surgical or medical) can be used. ${ }^{45}$

The first successful pregnancy in a woman with cystic fibrosis was reported in 1960. She delivered a healthy premature baby but died weeks postpartum from respiratory failure. Initial reports suggested high maternal and perinatal mortality and pregnancy generally was discouraged. In the subsequent four decades, however, the prognosis of cystic fibrosis has improved and attitudes towards pregnancy have gradually changed. There is an increasing trend for women with cystic fibrosis to undertake pregnancy. This can pose a challenge to the medical team but the emphasis now is on respecting women's autonomy and providing information, advice and support in helping them make decisions regarding pregnancy and motherhood. ${ }^{46}$

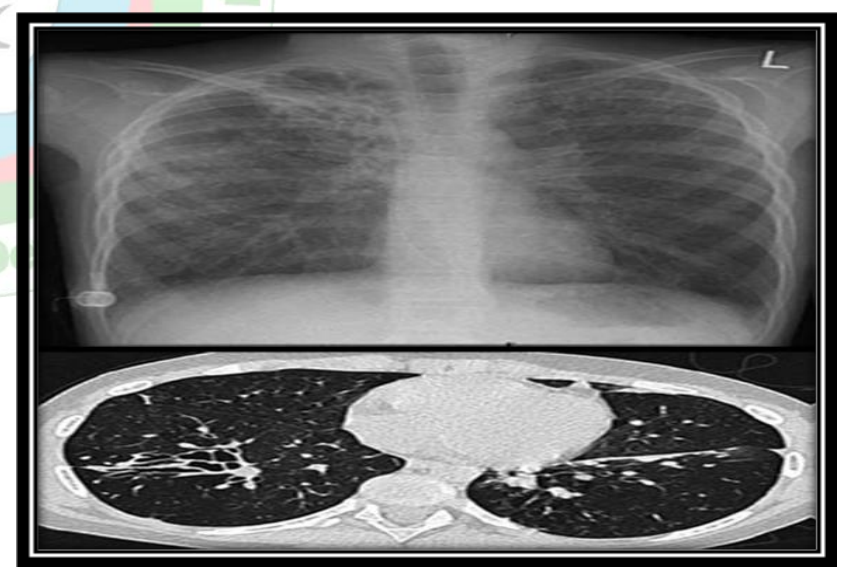

Figure 1: Severe bronchiectasis in end stage cystic fibrosis shown in chest radiograph (top) and computed tomogram (bottom). For reasons that are not fully understood, the upper lobes are often most severely affected, although the patient has severe bronchiectasis throughout the whole of the right lung. Note presence of indwelling intravenous catheter (a "port-a-cath") on the right lateral chest wall

\section{ETIOLOGY:}

Cystic fibrosis is an autosomal recessive disease. It is caused by mutations in the CFTR (cystic fibrosistrans membrane conductance regulator) gene on chromosome 7 that codes for a protein trans membrane conductance regulator (CFTR) protein which functions as a trans membrane cAMPactivated chloride channel. Both copies of the gene are mutated in clinical disease ${ }^{5,6}$. 
There are over 2000 different mutations in the CFTR gene that can cause disease. These mutations are divided into 5 classes:

- Defective protein synthesis

- Defective protein processing

- Disordered regulation

- Defective chloride conductance.

- Accelerated channel turnover

\section{CFTR mutations:}

Since the isolation of the CFTR gene there have been over 600 different mutations identified.6The most common of these, accounting worldwide for $68 \%$ of the mutant alleles, is $\ddot{A F 508, ~ a ~ t h r e e ~ b a s e ~ p a i r ~ d e l e t i o n ~ i n ~ e x o n ~} 10$ that removes phenylalanine 508 from the first NBD. Only a handful of others are common $(>1 \%$ of the total), but some are significantly enriched or depleted in specific ethnic populations. The vast majority are very rare, constituting a practical challenge to the clinical geneticist with respect to molecular diagnosis, but also a valuable epidemiological study which links protein structure to function and genotype to phenotype. All classes of mutation are observed, with the highest density of mutations occurring in the two nucleotide binding domains. known cystic fibrosis mutations occur in conserved regions suggesting conservation of function across species.

Mutations in CFTR may result in: (1) defective CFTR production, such as R553X, due to unstable mRNA and/or premature protein truncation, (2) defective processing of CFTR, such as ÄF508 or G480C, where the mutant protein is not processed to its mature glycosylated form and is not correctly localised to the apical membrane, but is retained in the endoplasmic reticulum and degraded. However, under permissive conditions in vitro, such as reduced temperature, correct localisation of mature protein can occur where it can function normally (in the case of G480C), or suboptimally (in the case of ÄF508), or (3) defective ion channel function, such as G551D or R117H, in which case some of the mutant protein becomes correctly localised but results in either very little residual function (in the case of G551D) or a substantially reduced level ofion transport (in the case of $\mathrm{R} 117 \mathrm{H})$. In each class of mutation the level of functional CFTR at the apical membrane of epithelial cells in patients with cystic fibrosis falls below a critical level, resulting in the characteristic clinical abnormalities observed in the organs in which CFTR is expressed.

The significant advances in our understanding of the disease and the development of new technologies now allows prenatal diagnosis, with diagnostic accuracy of $100 \%$ in almost all cases where an affected family member can be examined, and the implementation of carrier screening programmes to help individuals from affected families to make informed decisions in family planning. However, the picture is still complicated in a significant subset of unusual cases $^{51}$.

\section{PATHOPHYSIOLOGY:}

The CFTR protein actively transports chloride and bicarbonate toward the airway surface, secondarily bringing water with these ions. CFTR also affects other ion channels, most notably blocking the influx of sodium into the cell through the epithelial sodium channel. The CFTR abnormality has been shown to produce a number of changes in the airway, including acidification and decreased water and ion transit. This has led to large and wellrespected $\mathrm{CF}$ research groups to have different theories as to how CFTR produces airway disease. In the CF community, this controversy has been playfully referred to as the salt wars. The group at the University of North Carolina at Chapel Hill has staked their claim that CF pathogenesis is due to dehydration of the airway surface fluid, with a decreased osmotic drive for water transport into the airway lumen leading to collapse of the periciliary fluid layer in CF and a subsequent decrease in mucociliary transport ${ }^{7}$.This has led to the development of osmotic agents such as hypertonic saline and dry powder mannitol to attempt to restore the periciliary fluid layer. On the other side of this controversy, the CF research group in Iowa points out the importance of airway acidification as noted in 2 landmark articles published in the past year. An article published by this group demonstrates that airway acidification can initiate abnormalities in airway host defense in CF mice, and that host defense can be restored by restoring the $\mathrm{pH}$ balance $^{8}$.A second study from this group demonstrates that the acidic $\mathrm{pH}$ in the airway of both $\mathrm{CF}$ animal models and human airway cells from patients with $\mathrm{CF}$ will increase airway surface-liquid viscosity without changing airway mucin composition or volume. Furthermore, by increasing the $\mathrm{pH}$ of the airway surface liquid to a more neutral $\mathrm{pH}$, its viscosity will decrease to normal ${ }^{9}$.Studies such as these have led to active investigation into the evaluation of alkaline solutions such as bicarbonate as a potential therapy for CF airway disease. It is likely that there is truth in both of these theories, and that these complex interactions together lead to $\mathrm{CF}$ airway disease.

The altered CFTR protein in those with CF leads to viscous secretions that accumulate in the lungs. This gen-erates a cascade of pulmonary complications that starts with persistent coughing and leads to inflammatory states of bronchiectasis and, ultimately, respiratory failure (Fig-1). The mucus buildup also becomes a perfect milieu for persistent pulmonary infections. Patients with $\mathrm{CF}$ be-come infected early in life with Staphylococcus aureus (including methicillin-resistant Staphylococcus aureus) and Hemophilus influenzae, and later in life with Pseudomonas aeruginos $a^{10}$.Inhaled tobramycin and azithromycin are commonly used as antibiotic treatments ${ }^{11,12}$. 


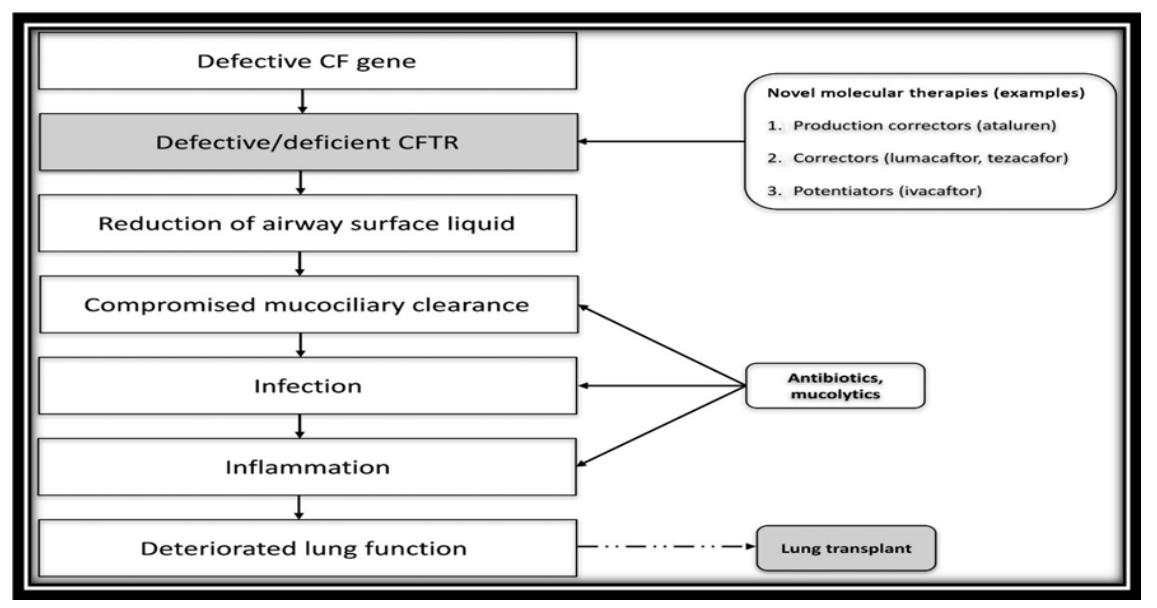

Figure 2: Pathology cascade for cystic fibrosis. The path physiological progression of CF With various types of Food and Drug Administration approved therapeutic strategies Modified from agent et al ${ }^{1,6}$

\section{RISK FACTORS:}

A pulmonary exacerbation of CF is usually identified by an increase in cough and sputum and a decrease in pulmonary function. This is treated with oral intravenous antibiotic therapy, either at home or in hospital. A systematic review compared in-patient intravenous antibiotics for a CF pulmonary exacerbation with out-patient intravenous therapy. Even using home intravenous therapy, hospitalbased therapy was more effective at restoring lung function ${ }^{13} \cdot \mathrm{A}$ similar review looked at the relationship of antibiotic treatment to recovery after an acute decline in pulmonary function, demonstrating that a "wait and see attitude" can lead to irreversible decline in pulmonary function, suggesting that all exacerbations with a decrease in $\mathrm{FEV}_{1}$ should be treated early and aggressively ${ }^{14}$. This was particularly true in subjects who had the best baseline lung function, and therefore the most to lose.

In CF subjects with normal lung function at baseline, there was dramatically better improvement with early institution of therapy. The odds ratio of recovery of $\mathrm{FEV}_{1}$ to baseline with hospital therapy compared with no intervention was 2.79; overall, in-patient treatment had a greater likelihood than out-patient therapy to lead to full recovery (odds ratio 1.94)

\section{CLINICAL FEATURES:}

Disease manifests in many organs, but most notably the upper and lower airways, pancreas, bowel, and reproductive tracts (table 1$)^{15}$.

Table 1: Age Related Presentations of Cystic fibrosis

\begin{tabular}{|l|l|l|}
\hline Age group & Common Presentations & Less common Presentation \\
\hline Antenatal & $\begin{array}{l}\text { Chronic villous sampling or amniocentesis in high risk } \\
\text { family; echogenic bowel on ultrasound }\end{array}$ & Perforated meconiumileus \\
\hline Neonatal & $\begin{array}{l}\text { Diagnosis made on newborn screening; meconium ileus } \\
(10 \% \text { of patients with cystic fibrosis) causing bowel } \\
\text { obstruction with or without perforation and peritonitis }\end{array}$ & $\begin{array}{l}\text { Gut atresias; obstructive jaundice ;fatsoluble vitamin } \\
\text { defeciencies (bleeding disorder, vitamin k; haemolytic } \\
\text { anaemia, vitamin E; raised intracranial ptressure, vitamin A) }\end{array}$ \\
\hline $\begin{array}{l}\text { Infants and young } \\
\text { children }\end{array}$ & $\begin{array}{l}\text { Recurrent respiratory symptoms (cough, wheeze, } \\
\text { pneumonias);failure to thrive (exocrine pancreatic } \\
\text { insufficiency present in 85-90\% of cases leads to } \\
\text { steatorrhoea, diarrhoea and abdominal distension) }\end{array}$ & $\begin{array}{l}\text { Rectal prolapsed; dehydration and electrolyte disturbance } \\
\text { (pseudo -Bartters syndrome);anaemia ,oedema and } \\
\text { hypoprotenaemia. }\end{array}$ \\
\hline
\end{tabular}

Adults with CF often present with exacerbations of one or more symptoms. Lung manifestations of CF include chronic bronchitis, abnormal pulmonary function tests, bronchiectasis, atypical asthma, allergic bronchopulmonary aspergillosis, and colonization with $P$. aeruginosa. Sinus manifestations of $\mathrm{CF}$ include chronic rhinosinusitis, chronic post-nasal drip, nasal polyposis, and panopacification of the paranasal sinuses. Pancreatic manifestations include pancreatic insufficiency, recurrent pancreatitis, and earlyonset diabetes. Hepatobiliary manifestations include focal biliary cirrhosis, cholelithiasis, periportal fibrosis, liver cirrhosis, portal hypertension, and variceal bleeding. Musculoskeletal manifestations include kyphoscoliosis, osteopenia/osteoporosis, and arthropathy. Hematologic manifestations include iron-deficiency anaemia or anaemia of chronic disease leading to splenomegaly. Nephrogenic manifestations include nephrolithiasis, nephrocalcinosis, hyperoxaluria, and hypocitraturia. Dermatologic manifestations include "salty sweat," digital clubbing, and cyanosis. Additional dermatologic conditions due to malabsorption include acrodermatitis enteropathica due to zinc deficiency and scaly dermatitis associated with fatty acid deficiency. Finally, males may be infertile due to an absent vas deferens while females have reduced fertility due to thickened cervical mucus ${ }^{17}$. 


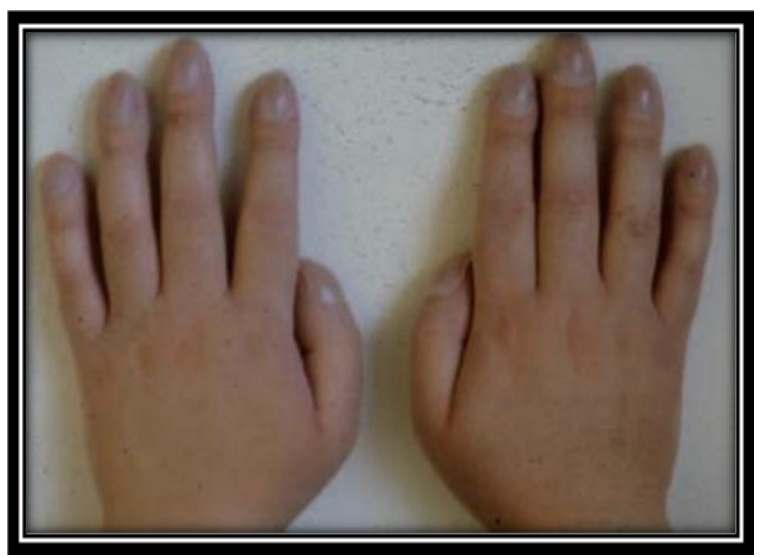

Figure 3: Finger clubbing indicates advance suppurative lung disease. It is not characteristic of asthma, with which older patients have sometimes been misdiagnose

\section{DIAGNOSIS:}

In the United States, newborns are screened for CF as part of a standard newborn screening panel. Some cases of CF are discovered on prenatal ultrasound which may demonstrate meconium peritonitis, bowel dilation, or absent gallbladder. Such findings often lead to prenatal CF carrier screening Fig $2^{19,20}$.

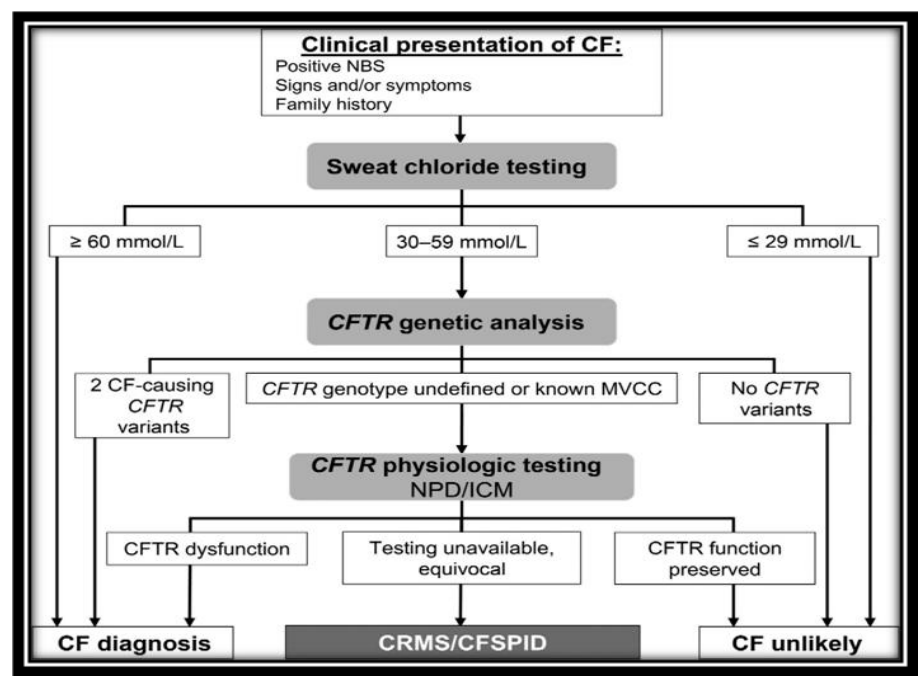

Figure 4: Updated testing algorithm for CF diagnosis. Evidence of disease and CFTR dysfunction.

To diagnose $\mathrm{CF}$, the following criteria must be met:

\section{Suspicion for Cystic Fibrosis}

- Sibling with cystic fibrosis

- Positive newborn screen

- Clinical symptoms consistent with CF in 1 or more organ systems

- Chronic sinopulmonary disease

- Gastrointestinal or nutritional abnormalities

- Salt loss syndromes

- Obstructive azoospermia

\section{Evidence of CFTR Dysfunction}

- Elevated sweat chloride 2 than $60 \mathrm{mEq} / \mathrm{L}$ on two occasions

- Two disease-causing CFTR mutations

- Abnormal nasal potential difference

The diagnostic pathway starts with a sweat chloride test. If normal but still symptomatic, a repeat sweat chloride test is indicated. If the test is abnormal, DNA testing is indicated. If 1 or less CFTR mutations are found, expanded DNA analysis is indicated. However, the finding of 2 CF-related mutations confirms the diagnosis of cystic fibrosis.

The test for immunoreactive trypsinogen (IRT), a pancreatic enzyme, increases sensitivity and specificity in screening newborns with meconium ileus for CF. IRT monitoring can be correlated with the severity of $\mathrm{CF}$ and when it drops below detectable levels can indicate the need to start pancreatic enzyme replacement.

Additional diagnostics may be indicated depending on the presenting symptoms. A chest $\mathrm{x}$-ray may help identify hyperinflation, bronchiectasis, abscesses, or atelectasis. Sinus radiography may demonstrate panopacification of the paranasal sinuses. Abdominal radiology may be helpful in neonates who present with meconium ileus. Broncho alveolar lavage typically shows many neutrophils, and microbiology is commonly positive for Haemophilus influenza, Staphylococcus aureus, Pseudomonas 


\section{aeruginosa,}

Burkholderiacepacia,

\section{Escherichia}

coli, or Klebsiella pneumonia.

Pulmonary function testing is a major tool for evaluating and monitoring disease state and progression in CF. Spirometry is the commonly used pulmonary function test. It measures the volume of air exhaled during a forceful and complete exhalation after a maximal inhalation. The total exhaled volume, known as the forced vital capacity (FVC), the volume exhaled in the first second, known as the forced expiratory volume in one second (FEV1), and their ratio (FEV1/FVC) are the most important variables reported. These values allow for interpretation of the status of the lung ventilation function. These values are compared to an expected normal for age, height, and gender to generate an expected normal value. The measured value is then calculated as a percent of normal where normal equals $100 \%$. A normal or high FEV1 and/or a low FVC may indicate restrictive lung disease. A low FEV1 with a high FVC indicate obstructive lung disease with airway trapping. Cystic fibrosis can be expected to show air trapping patterns with low FEV1 values proportional to the severity of the disease $^{21}$.

Several methods of newborn screening may be implemented to detect potential $\mathrm{CF}$, such as the immunoreactivity trypsinogen test (IRT), double IRT testing, and pancreatitisassociated protein testing. A positive or equivocal screening test should be followed by CFTR genetic testing and the sweat chloride test, which involves the collection of sweat after the transdermal administration of pilocarpine in order to confirm the diagnosis ${ }^{41}$. Sweat chloride testing is the gold standard for discriminating between true positive and false positive results in newborns ${ }^{40,42}$.

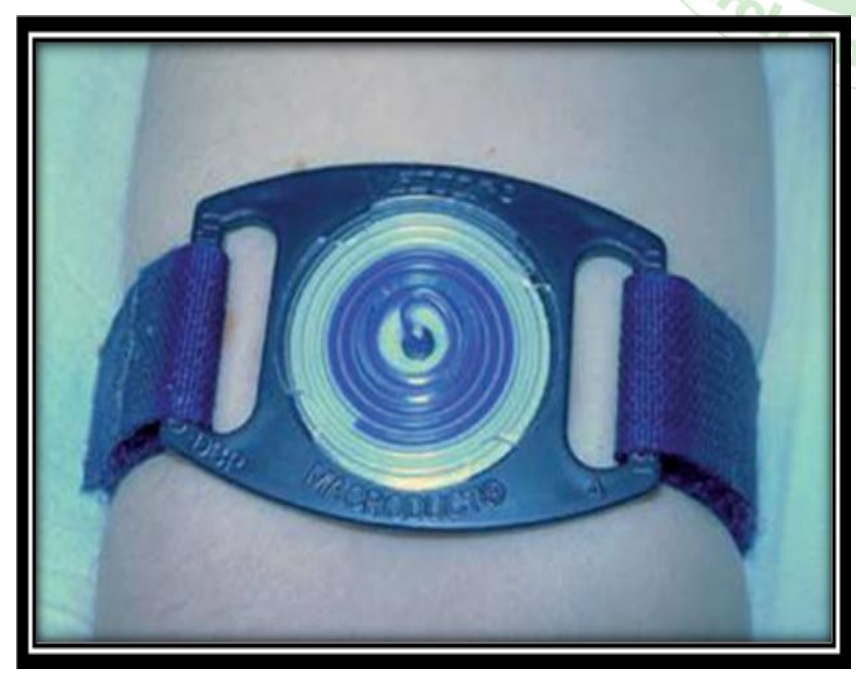

Figure 5: Child's arm during sweat test with the macroduct system. After pilocarpine iontophoresis to stimulate sweating, the closed capillary collecting system is applied to the skin of the forearm. Sweat can be seen entering the tubing (blue); electrolyte analysis can be reliably performed on as little as $50 \mu 1$ of sweat

\section{COMPLICATIONS:}

Respiratory system complications include Bronchiectasis, Chronic infections leading to pneumonia, growths (nasal polyps), hemoptysis, pneumothorax and eventually respiratory failure. Digestive system complications include nutritional deficiencies including fat and fat soluble vitamins and diabetes (Nearly $20 \%$ of people with cystic fibrosis develop diabetes by age 30). Additionally, progressive hepatic dysfunction, gallstones, intestinal obstruction, intussusception, small intestine bacterial overgrowth (SIBO) and distal intestinal obstruction syndrome (DIOS) may also manifest. Other complications may include Infertility, Osteoporosis, Electrolyte imbalances and dehydration manifesting as increased heart rate, fatigue, weakness and low blood pressure. Because of better quality medical interventions and comprehensive care, there is a remarkable increase in percentage of patients $(29.2 \%$ in 1986 to $49.7 \%$ in 2013) surviving above the age of 18 years $^{23}$.

\section{TREATMENT:}

\section{Pulmonary Therapy:}

\section{Non Pharmacological Treatment:}

Airway clearance therapy is performed on a daily basis to improve clearance of mucus from the lungs. Postural percussion therapy or chest physiotherapy devices are routinely used by patients to loosen and clear mucus. Positive expiratory pressure devices help keep the patient's airway open. Breathing out with moderate force through resistance allows airflow to get beneath mucus obstruction so that it can be coughed out ${ }^{43}$. See Table 2 for a detailed list of airway-clearance therapies.

Table 2: Non Pharmacological Airway Clearance Therapy

\begin{tabular}{|l|l|}
\hline Airway Clearance Therapy & Outcome \\
\hline Percussion and postural drainage & Listens and clears mucus \\
\hline Positive expiratory pressure & Open airways \\
\hline Active cycle of breathing technique & $\begin{array}{l}\text { Relaxes airways and clears } \\
\text { mucus out of lungs }\end{array}$ \\
\hline Autogenic drainage & $\begin{array}{l}\text { Moves mucus out of the } \\
\text { lungs }\end{array}$ \\
\hline Physical activity (exercise) & Improves lung function \\
\hline
\end{tabular}

\section{Pharmacological Treatment:}

Drugs to treat $\mathrm{CF}$ are designed to improve the clearance of mucus from the lungs and to treat persistent infections. The most recent guidelines from the Cystic Fibrosis Foundation regarding medication use were published in 2013.Previous guidelines were updated and two new medications were evaluated and graded using the United States Preventive Services Task Force definitions.The Cystic Fibrosis Foundation recommends the following treatments as having a high certainty of substantial net benefit, grade A, for moderate-to-severe disease: inhaled tobramycin, dornasealfa, ivacaftor, and inhaled aztreonam.see table3 complete list of drug products with a high/substantial or high/moderate grade recommendation ${ }^{43}$. 
Table: 3 complete list of drug products with a high/substantial or high/moderate grade recommendation

\begin{tabular}{|c|c|c|}
\hline Drug & Dose & Common side effects \\
\hline Inhaled hypertonic saline & $4 \mathrm{~mL} /$ dose via oral inhalation twice daily & $\begin{array}{l}\text { Cough, bronchospasm, pharyngitis, hemoptysis, } \\
\text { sinusitis, sneezing }\end{array}$ \\
\hline Dornase alfa & $2-5 \mathrm{mg}$ oral inhalation ,1-2 times daily & $\begin{array}{l}\text { Chest pain, conjunctivitis, pharyngitis, } \\
\text { hoarseness/voice alterations }\end{array}$ \\
\hline Azttreonam & $75 \mathrm{mg}$ nebulized 3 times daily for 28 days & $\begin{array}{l}\text { Cough, nasal congestion, wheezing } \\
\text {,pharyngolaryngeal pain, chest discomfort, } \\
\text { bronchosnasm }\end{array}$ \\
\hline Tobramycin & $300 \mathrm{mg}$ bf for 28 days & $\begin{array}{l}\text { Nephrotoxicity, ototoxicity, cough, pharyngitis, } \\
\text { rhinitis dyspnea, asthma }\end{array}$ \\
\hline Azithromycin & $250 \mathrm{mg}$, po 3 days per week & Diarrhoea ,nausea, vomiting \\
\hline Ivacaftor & $150 \mathrm{mg}$ po & $\begin{array}{l}\text { Abdominal pain, diarrhoea, nausea, pharyngitis, rash, } \\
\text { headache, nasal congestion }\end{array}$ \\
\hline Ibuprofen & $20-30 \mathrm{mg} / \mathrm{kg}$ and ,max dose $3200 \mathrm{mg} /$ day & Abdominal pain constipation edema, neutropenia \\
\hline
\end{tabular}

Hypertonic Saline: Inhaled hypertonic saline is administered by nebulization to restore airway hydration, induce expectoration of sputum, and enhance mucociliary function. Although $7 \%$ is the standard strength, it can be administered in a strength as low as $3 \%$ for patients experiencing side effects, which may include cough, sore throat, and chest tightness. Chronic use of inhaled hypertonic saline is recommended for patients aged 6 years and older for improved lung function and quality of life, and reduced exacerbations ${ }^{43}$.

Dornasealfa (Pulmozyme): This is a recombinant human deoxyribonuclease. It cleaves to the extracellular DNA of mucus and decreases the viscosity of mucus, making it easier to cough up. This medication is administered by nebulization route one to two times daily. For patients aged 6 years and older with moderate-to-severe disease, it has been shown to reduce exacerbations while also improving lung function and quality of life ${ }^{43}$.

Antibiotics: Bacterial colonization and growth in the lungs often causes pulmonary exacerbations in $\mathrm{CF}$ patients. Common characteristics of pulmonary exacerbations include increased coughing, diminished pulmonary function, and an increase in mucus production with a change in color.Acute exacerbation treatment frequently requires inpatient hospital treatment unless the home can provide a level of IV care equal to a hospital setting. Patients experiencing an acute exacerbation should stay on their chronic therapy along with an intensified airway clearance treatment. Evidence is lacking to recommend using one versus multiple antibiotics, or the optimal duration of treatment.

In patients (aged $\geq 6$ years) with chronic presence of Pseudomonas aeruginosa ( $P$ aeruginosa) in airway cultures, prophylactic use of antibiotics (tobramycin, aztreonam, and azithromycin) is recommended. The use of inhaled tobramycin and aztreonam delivers the drug locally to the lung and decreases the risk of systemic side effects. For chronic use, tobramycin is administered by nebulization (300 mg) or dry powder inhalation $(112 \mathrm{mg}$ ) twice daily for 28 days on and 28 days off. It can also be administered IV or
IM at a dose of $10 \mathrm{mg} / \mathrm{kg} /$ day given in four equally divided doses. However, these routes of administration are not included in the guidelines for chronic use.Aztreonam inhalation solution is an alternative antibiotic for patients with chronic colonization of $P$ aeruginosa. Although indicated for this use, it is considered to be an orphan drug for this purpose.

Aztreonam $75 \mathrm{mg}$ is administered by nebulization route three times daily for 28 days on and then 28 days off. If a patient is chronically using a bronchodilator and mucolytic, the bronchodilator should be used first, followed by the mucolytic, and then the aztreonam. In a head-to-head clinical trial, aztreonam inhalation solution was compared with inhaled tobramycin and demonstrated clinical superiority in improving lung function.Although approved for IV use from age 9 months, it is not included in the guidelines for chronic use.Azithromycin is an oral agent recommended by the guidelines for use with chronic $P$ aeruginosa to improve lung function and reduce exacerbations. However, it is considered to have a slightly lower estimated end benefit when compared with inhaled tobramycin and aztreonam.Evidence for the chronic use of other antibiotics is inconclusive regarding improvement in lung function and quality of life, and reduced exacerbations. ${ }^{43}$

CFTR Modulator Therapies: In a recently published guideline on CFTR modulator therapy, 30 different treatment recommendations were made based on gene mutation. Ivacaftor (Kalydeco) was the first approved disease-modifying (potentiator) drug for CF (2012) compared with the previous drugs that only addressed the treatment of symptoms. In one study, over a 48-week period it was reported to improve lung function along with several other CF-related problems. Ivacaftor is approved to treat a total of 38 CFTR gene mutations. The Cystic Fibrosis Foundation guidelines recommend chronic use of ivacaftor in patients aged 2 years and older with at least one CFTR 
mutation for improvement in lung function and quality of life, and reduced exacerbations. It is available in tablet and oral granules formulations. A disadvantage of ivacaftor is that it only targets a small portion $(5 \%)$ of $\mathrm{CF}$ patients with a G551Dmutation.

Lumacaftor $(200 \mathrm{mg})+$ ivacaftor $(125 \mathrm{mg})$, Orkambi, is the first approved (2015) CFTR corrector and potentiator combination therapy. It is approved for use in CF patients with two copies of the Phe508del (formerly F508del) CFTR mutation who are aged 6 years and older. Approximately $45 \%$ of the CF population in the U.S. could benefit from this therapy. Orkambi showed improvement in pulmonary function and reduction in pulmonary exacerbation compared with placebo in two phase III clinical trials. Patients should be counseled to take CFTR modulators with fat-containing food.

Anti-inflammatory Medications: The Cystic Fibrosis Foundation recommends against the chronic use of inhaled or oral corticosteroids and leukotriene modifiers owing to insufficient evidence that they can improve lung function and quality of life, or reduce exacerbations. Chronic use of oral high-dose ibuprofen to reach a peak plasma concentration of $50-100 \mathrm{mcg} / \mathrm{mL}$ is recommended for individuals aged 6 to 17 years with an $\mathrm{FEV}_{1}$ greater than $60 \%$ predicted in order to slow the progressive loss of lung function in this group. There is insufficient evidence for use of ibuprofen in patients aged 18 years or older.

Other Chronic Treatments: There are several potential chronic medications that have neither a positive nor negative recommendation for use in patients aged at least 6 years owing to lack of evidence, according to the Cystic Fibrosis Foundation guidelines. Examples of such chronic medications include inhaled beta2 adrenergic agonists, leukotriene modifiers, inhaled or oral $\mathrm{N}$-acetylcysteine or inhaled glutathione, and inhaled anticholinergics ${ }^{43}$.

\section{GIT:}

Preventing or treating intestinal blockages - oral rehydration and osmotic laxatives (incomplete blockage) and hyperosmolar contrast enemas (complete DIOS). A balanced electrolyte intestinal lavage solution or enema containing (diatrizoate,meglumine and diatrizoate sodium) depending on vomiting status ${ }^{31}$. To prevent recurrence, regular administration of oral polyethylene glycol 3350 may be given for 6 months to 1 year. Pancreatic insufficiencypancreatic enzyme replacement therapy (PERT) containing multiple combinations of proteases, lipases and amylases ${ }^{32}$.

\section{Nutritional Support:}

Management of CF requires good nutrition and appropriate supplementation of vitamins and pancreatic enzymes. The patient's underlying gene mutation, increased effort of breathing, and the metabolic consequences of chronic infection require increased nutritional intake. The recommended increase in energy intake ranges from $110 \%$ to $200 \%$ of that needed for healthy individuals. ${ }^{35}$ Improved pulmonary function and survival is associated with normal weight ranges in $\mathrm{CF}$ patients. ${ }^{35}$ It is recommended that patients with pancreatic insufficiency consume a highcalorie diet with appropriate unrestricted fat intake. High-fat diets (40\% of total calories) promote growth and improve lung function ${ }^{36}$.

Pancreatic enzyme replacement therapy (PERT) and fatsoluble vitamin supplementation are used to treat pancreatic insufficiency and improve fat absorption. Because of the inability to absorb fat-soluble vitamins $\mathrm{A}, \mathrm{D}, \mathrm{E}$, and $\mathrm{K}$, patients with pancreatic insufficiency should be monitored and given prophylactic supplementation of fat-soluble vitamins. The current recommendation for PERT from the American Society for Parenteral and Enteral Nutrition is to start with 500 lipase units per kilogram of body weight per meal up to a maximum of 2,500 units $/ \mathrm{kg} / \mathrm{meal}$ or 10,000 units/kg/day ${ }^{36}$. The Cystic Fibrosis Foundation guidelines recommend starting with a low dose of 500 units per gram of dietary fat ingested per day up to 4,000 units per gram ${ }^{37}$. The patient's clinical response should be used to determine the dosing titration regardless of which recommendation or guideline is followed.

Pancreatic enzyme products utilize enteric-coating dosage forms because of their sensitivity to an acidic environment. Histamine-2 receptor antagonists and proton pump inhibitors are sometimes used to improve absorption of pancreatic enzymes by maintaining an alkaline $\mathrm{pH}$, especially when the enzyme dosage reaches maximum levels ${ }^{34,37}$. However, the current evidence concerning efficacy is equivocal ${ }^{38,39}$. Patients should be counseled not to crush or chew pancreatic-enzyme capsules. The capsules may be opened and sprinkled on soft, nonalkaline foods such as apple sauce $^{36}$. A mouth rinse after use helps to prevent ulceration. Pancreatic enzymes should be administered prior to consuming meals, snacks, and fat-soluble vitamins. Patients receiving dosages above these guidelines may develop fibrosing colonopathy ${ }^{34,36,37}$. fig 6 


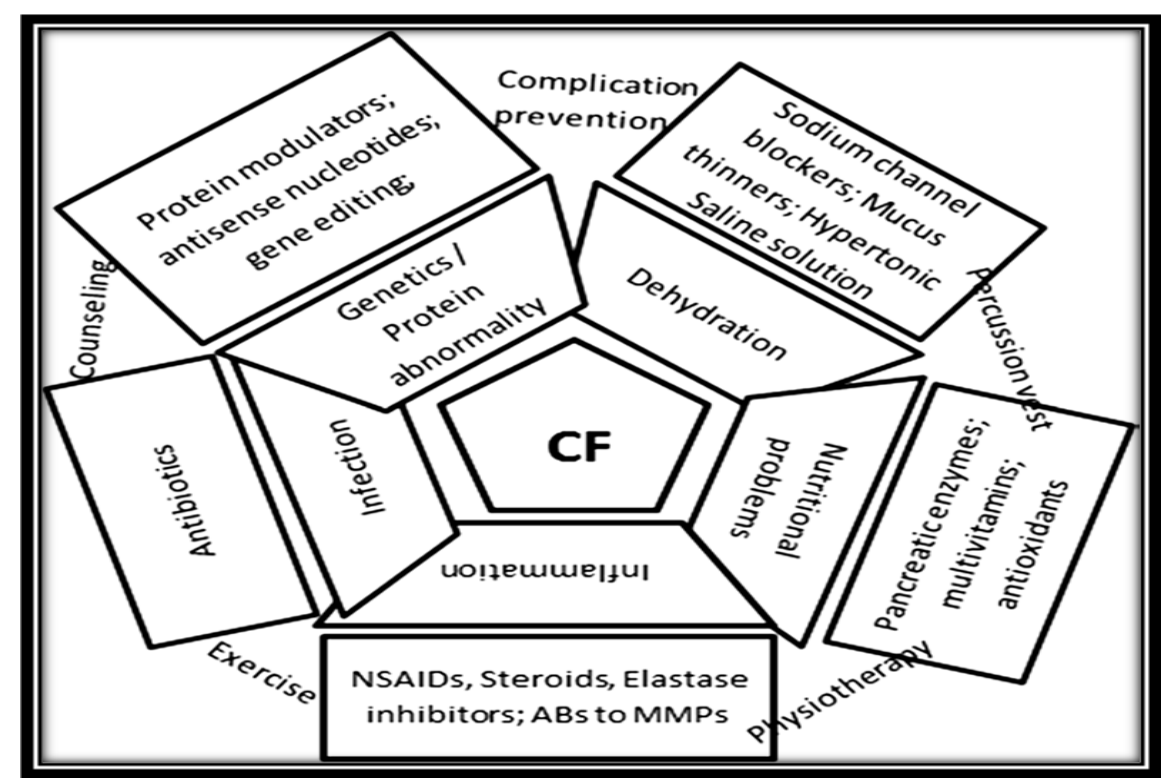

Figure 6: The main pathophysiological dysfunctions and treatment modalities for CF patients. Inner trapezoid boxes depict the pathophysiological abnormalities and outer rectangular boxes depict the main treatments. The texts connecting the outer boxes show nonpharmacologicalmanagement ${ }^{33}$

\section{Effect of Cystic Fibrosis on Pregnancy and Breast Feeding:}

Approximately 30-40 women with cystic fibrosis undertake pregnancy each year in the UK .Early reports suggested that pregnancy was associated with high rates of miscarriage, premature delivery and stillbirth .Recent data, however, demonstrate good fetal outcomes. The proportion of live births is now $70-90 \%$ and the spontaneous miscarriage rate is no higher than in the general population. The prematurity rate is approximately $25 \%$ but varies between studies from 8 $46 \%$.Some women are delivered preterm because of deteriorating maternal health, although women with poorer lung function have higher rates of spontaneous preterm labour. There have been no reports of higher rates of congenitalmal formations in babies born to mothers with cystic fibrosis. The average weight gain in pregnancy for healthy women is $10-12 \mathrm{~kg}$. The additional calorific requirements of pregnancy can be difficult to achieve for women with cystic fibrosis and pancreatic insufficiency. The reported average weight gain in term pregnancies varies from $5.5-10.3 \mathrm{~kg}$. A low body mass index prior to conception increases the incidence of miscarriage and premature delivery. Babies born to women with cystic fibrosis are, in general, appropriately grown for their gestational age. Pre-existing and gestational diabetes are more common in pregnancies in women with cystic fibrosis (incidence 14-20\%) and increase the risk of pregnancy complications, including preterm delivery and stillbirth. The mode of delivery depends on obstetric indications, maternal health and gestational age. In the study by Edenboroughet al., $31 \%$ were delivered by caesarean section. Two-thirds of these were preterm deliveries and the remainder were for obstetric indications at term. Of the women who had vaginal deliveries, $40 \%$ required instrumental delivery ${ }^{47,48}$.
Drugs during Pregnancy and Breastfeeding in Cystic Fibrosis Patients:

\section{Respiratory System:}

Antibiotics:

Pregnancy:

Antibiotics are the major components of CF treatment and are administered chronically (e.g. inhaledantibiotics, macrolides used for their immunomodulatory properties) or intermittently to prevent, eradicate, control or treat respiratory infections. Due to increased antibiotic clearance and distribution, higher doses are usually administered to $\mathrm{CF}$ patients, and a combination of antibiotics is often necessary due to the presence of multiple or multiresistant microorganisms. Most antibiotics are safe for use in pregnancy. All the antibiotics for which the placental transfer has been studied can be detected in the amniotic fluid. Penicillins, well-documented cephalosporins (e.g. cefaclor, cefuroxim, ceftriaxon) and macrolides (azithromycin, clarithromycin, erythromycin) are first-line treatments during pregnancy for sensible pathogens such as Staphylococcus aureus or Haemophilus influenzae.Antipseudomonalpenicillins, including piperacillin,piperacillin-tazobactam, temocillin, and less documented cephalosporins (ceftazidime, cefepime), carbapenems(meropenem, imipenem, ertapenem), monobactam (aztreonam), can be safely used intravenously for Pseudomonas exacerbation.Clindamycin, sulfonamides, trimethoprim, and cotrimoxazoleare antibiotics of second choice in pregnancy, and can be used when first-line antibiotics are ineffective or in case of allergy .

In case of long-term first trimester use of sulfonamides with a folate antagonist(e.g. trimethoprim), increased folic acid intake ( $1-5 \mathrm{mg} / \mathrm{day})$ is recommended, even though evidence 
is lacking to support its efficacy in preventing teratogenic effects. When sulfonamides are used until birth, a rise in bilirubinmay be observed, especially in premature infants. Tetracyclines have not been associated with an increasedrisk of malformations However, they cancause tooth discoloration and inhibition of bone growth if used after the 15th week of pregnancy due to binding to calcium ions . Aminoglycosides carry a small risk of fetalnephro- and ototoxicity Therefore, their intravenous use should be reserved for life-threatening infections. Dose adjustment by therapeutic drug monitoring in pregnant $\mathrm{CF}$ patients is recommended with gentamicin, amikacin and tobramycin for efficacy and safety optimization in pregnancy Inhaled tobramycin is associated with minimal risks because of the limited systemic absorption. Quinolones have been associated with irreversible damage of joint cartilages in young animals treated directly (i.e. none resulting from in utero exposure). This has never been observed after in utero exposure to quinolones in humans. In the absence of alternatives, the well-documented norfloxacin and ciprofloxacin should be preferred. Because of the lack of security data in pregnancy, intravenous colistin should be reserved for life-threatening infections, while its limited systemic absorption after inhalation limits the risks ${ }^{48}$.

\section{BREAST FEEDING:}

Most antibiotics are excreted into breastmilk in very small amounts . A transient effect on the infants' stool consistency is the most frequently observed adverse event in infants exposed to antibiotics via breast milk. Antibiotics discussed below are compatible with breastfeeding. Penicillins, cephalosporins and macrolides are first-line treatments during lactation A short-time therapy with tetracyclines is possible $(<3$ weeks), while clindamycin, sulfonamides, colistinand quinolones should only be used in CF lactating womenwhen first-line antibiotics are ineffective. As a result of their poor oral bioavailability, carbapenems, monobactams or aminoglycosides are probably safe for the breastfed infant after maternal intravenous administration. Inhaled use of antibiotics is compatible with breastfeeding ${ }^{48}$.

\section{ANTIFUNGALS AND ANTIVIRALS:}

Although invasive fungal infections are infrequent in the non transplanted $\mathrm{CF}$ patients, sensitization to fungi and notably to aspergillus may lead to allergic bronchopulmonary aspergillosis. The latter necessitates treatment with systemic corticosteroids, which may be combined with antifungal agents .Respiratory viral infections may lead to prolonged symptoms and may worsen the course of CF. Despite a paucity of studies in this context, it is considered that neuraminidase inhibitors against influenza infection may be helpful in limiting clinical manifestations.

\section{GASTROINTESTINAL SYSTEM:}

\section{Pancreatic Enzymes:}

Pregnancy: No data of pancreatic enzyme use during pregnancy are available, and therefore proper risk assessment is not possible to date. As those preparations have a very limited systemic absorption, no fetaladverse effects are expected.

Breastfeeding: Pancreatic enzymes can be considered compatible with breastfeeding due to their limitedabsorption ${ }^{48}$.

\section{ANTACIDS:}

Pregnancy: Antacids may be used throughout pregnancy. Fixed combinations of aluminium and magnesium salts should be preferred and used at recommended doses. No associations with an increased rate of birth defects have been detected after more than 1,500 exposures during the first trimester with ranitidine. No associations have been found between proton pump inhibitor use during pregnancy and increased rate of birth defects in studies including several thousands of patients. Omeprazole, the best investigated proton pump inhibitor to date, and its enantiomer esomeprazole are the medication of choice. Misoprostol has an uterotonic activity and is linked to an increased risk of Moebius sequence. It is contraindicated during pregnancy except for specific obstetrical indications 48 .

Breastfeeding: Antacids can be used during breast feeding. Ranitidine reaches variable concentrations in the mother's milk, corresponding however to smaller amounts than the usual pediatric dosage. Omeprazole and esomeprazole are acid labile compounds and are likely to be destroyed in the infant's stomach prior to systemic absorption. Based on these considerations, antacids are not expected to cause adverse effects in breastfed infants.

\section{PROKINETICS:}

Pregnancy: Metoclopramide has been widely used in the treatment of nausea and vomiting in pregnancy, and safety data are numerous for this compound. It is considered as the antiemetic of choice during pregnancy. Domperidone is not a drug of choice in pregnancy as its safety has only been demonstrated in a small study.

Breastfeeding: Metoclopramide and domperidone are excreted in small amounts into breast milk with no adverse events reported in breastfed infants. If both prokinetics may be used during breastfeeding, domperidone is excreted in smaller amounts into milk and is less able to cross the infant blood-brain barrier $^{48}$.

\section{VITAMINS}

Pregnancy: Approximately $85 \%$ of $\mathrm{CF}$ patients present exocrine pancreatic insufficiency, which predisposes to an 
impaired absorption of fat-soluble vitamins. Adequate nutritional status has to be maintained throughout pregnancy. Serum levels of vitamins that are at high risk of deficiency [i.e. fat-soluble vitamins (A, D, E, K) and vitamin B 12 should be closely monitored. A teratogenic effect similar to retinoids has been associated with high doses of vitamin A (>25,000 UI). This risk has not been confirmed in a large cohort study evaluating the risk of high doses of vitamin A. A daily dose of $<10,000 \mathrm{UI}$ is considered safe. Supplementation with folic acid (0.4-0.8 mg daily) should be started in women planning pregnancy and continued throughout the first trimester for the prevention of neural tube defects ${ }^{48}$.

Breastfeeding: Adequate nutritional status has to be maintained in CF patients throughout lactation. Serum levels of vitamins that are at high risk of deficiency [i.e. fat-soluble vitamins $A, D, E, K$ ) and vitamin B 12] should be closely monitored $^{4}$.

R 2 major roles in CF management: ensuring the effectiveness of medication therapy and facilitating easy access to prescribed regiments. There is an urgent need to increase pharmacist-provided outpatient care for people with CF to improve adherence, medication self-management, and overall health-related quality of life. As with many chronic diseases, a dedicated multidisciplinary team may help to enhance CF treatment management and outcomes. Specialty pharmacy programs may help to provide patients with $\mathrm{CF}$ with comprehensive care. More than one-half of all people living with $\mathrm{CF}$ are older than 18 years, so it is essential that younger patients develop self-management as they make the transition from adolescence to adulthood. Adolescents with CF are particularly vulnerable as they approach adulthood; they are already among those with the highest rates of non adherence, and at this age they are expected to assume greater responsibility to manage complex medication regimens. Previous studies have found that a standardized transition program with pediatric and adult CF programs working closely together needs to be implemented to help ease the transition into adulthood for adolescents with $\mathrm{CF}$. Pharmacists as medication experts are well equipped to address drug therapy problems and provide other outpatient clinical services for young people with $\mathrm{CF}$ as they gain more self-management responsibility. A CF center in England implemented a dedicated $\mathrm{CF}$ pharmacist and after 2 years

\section{REFERENCES:}

1. Poncin W, Lebecque P. Lung clearance index in cystic fibrosis. Rev Mal Respir. 2019; 36(3):377-395.

2. Lyamin AV, Ismatullin DD, Zhestkov AV, Kondratenko OV. The laboratory diagnostic in patients with mucoviscidosis: A review. Klin. Lab. Diagn. 2018; 63(5):315-320.

3. Eschenhagen P, Schwarz C. Patients with cystic fibrosis become adults: Treatment hopes and disappointments. Internist (Berl). 2019; 60(1):98108. observed benefits such as improved medication monitoring, communication with the multidisciplinary care team, and efficient use of resources with the goal of reducing care costs. Pharmacists can also assist patients with medication access. An stead et al. demonstrated that $52 \%$ of their adult patients with CF were unable to access 2 or more of the medications prescribed by their provider. Ninety-eight percent of these patients were able to access their prescribed medications when a pharmacist and pharmacy technician were included as CF health care team members.In addition, patients with $\mathrm{CF}$ have to use multiple payers (primary and secondary insurances) and different types of pharmacies, which commonly include specialty and mail-order pharmacies. Insurance policies also dictate certain pharmacies that can be used, based on contracts, which can cause delays for patients to receive their medications, because not all pharmacies stock common CF medications, such as supplements and Pulmozyme. Many patient assistance programs exist to aid in insurance coverage for patients with CF. Pharmacists can help in navigating insurance coverage challenges to ensure that patients with $\mathrm{CF}$ have access to their medications and receive them in a timely manner ${ }^{50}$.

\section{CONCLUSION:}

Cystic fibrosis is autosomal recessive disease. The predicted age of survival for people with cystic fibrosis is early 40's.Life expectancy is mostly affected by the severity of disease, age of diagnosis and the type of cystic fibrotic gene mutation. Approaches like gene transfer, search for alternative targets and disease modeling are needed.

\section{ABBREVIATIONS:}

- CF
- CFTR
-
- cAMP
- DIOS
- SIBO
- DNA
- FEV
- IRT
- FEV
- PERT
- GIT

: Cystic Fibrosis

: Cystic fibrosis transmembrane conductace regulator

: Cyclic Adenosine 3,5 monophosphate

: Distal Intestinal Obstruction Syndrome

: Small Intestine Bacterial Overgrowth

: Deoxyribonucleic acid

: Forced expiratory volume

: Immunoreactive trypsinogen

: Forced vital capacity

: Pancreatic enzyme replacement therapy

: Gastrointestinal tract 
7. Boucher RC. Evidence for airway surface dehydration as the initiating event in CF airway disease. J Intern Med 2007; 261(1):5-16.

8. Shah VS, Meyerholz DK, Tang XX, Reznikov L, AbouAlaiwa M, Ernst SE, Karp PH, et al. Airway acidification initiates host defense abnormalities in cystic fibrosis mice. Science 2016; 351(6272):503507.

9. Tang XX, Ostedgaard LS, Hoegger MJ, Moninger TO, Karp PH, Mc Menimen JD, et al. Acidic pH increases airway surface liquid viscosity in cystic fibrosis. J Clin Invest 2016; 126(3):879-891.

10. Osenfeld M, Ramsey BW, Gibson RL. Pseudomonas acquisition in young patients with cystic fibrosis: patho-physiology, diagnosis, and management. Curr Op in Pulmonol Med 2003; 9:492-7.

11. Ramsey BW, Pepe MS, Quan JM, Otto KL, MontgomeryAB, Williams-Warren J, et al. Cystic Fibrosis Inhaled To-bramycin Study Group. Intermittent administration of inhaled tobramycin in patients with cystic fibrosis. NEngl J Med 1999; 340:23-30.

12. Saiman L, Marshall BC, Mayer-Hamblett N, Burns JL, Quittner AL, Cibene DA, et al. Macrolide Study Group. Azithromycin in patients with cystic fibrosis chronically infected with Pseudomonas aeruginosa: a randomized controlled trial. JAMA 2003; 290:1749-56.

13. Bryant PA, Katz NT. Inpatient versus outpatient parenteral antibiotic therapy at home for acute infections in children: a systematic review. Lancet Infect Dis 2017; (17):30345-30346.

14. Morgan WJ, Wagener JS, Pasta DJ, Millar SJ, VanDevanter DR, Konstan MW; Scientific Advisory Group, Investigators, and Coordinators of the Epidemiologic Study of Cystic Fibrosis. Relationship of antibiotic treatment to recovery after acute FEV1 decline in children with cystic fibrosis. Ann Am ThoracSoc2017; 14:937-942

15. Davis PB. Cystic fibrosis since 1938. Am J Respir Crit Care Med 2006; 173:475-82.

16. Agent P,Parrott H. Inhaled therapy in cystic fibrosis: agents, devices and regimens. Breathe 2015; 11:110-8.

17. Elaine Yu Sandeep Sharma. Cystic Fibrosis Treasure Island (FL): Stat Pearls Publishing; 2019.

18. Farrell PM, White TB, Ren CL, Hempstead SE, AccursoF, Derichs N, et al. Diagnosis of cystic fibrosis: consensusguidelines from the Cystic Fibrosis Foundation. J Pedi-atr 2017; 181:S4-15.

19. Pawlaczyk-Kamieńska T, Borysewicz-Lewicka M, Śniatała R, BaturaGabryel H, Cofta S. Dental and periodontal manifestations in patients with cystic fibrosis - A systematic review. J. Cyst. Fibros. 2018; 22.

20. Radovanovic D, Santus P, Blasi F, Sotgiu G, D'Arcangelo F, Simonetta E, Contarini M, Franceschi E, Goeminne PC, Chalmers JD, Aliberti S. A comprehensive approach to lung function in bronchiectasis. Respir Med. 2018 Dec; 145:120-129.

21. PallinM. Cystic fibrosis vigilance in Arab countries: The role of genetic epidemiology. Respirology. 2019; 24(2):93-94.

22. Rosenstein BJ, Cutting GR, for the Cystic Fibrosis Foundation Consensus Panel. The diagnosis of cystic fibrosis: a consensus statement. J Pediatr1998; 132:589-95.

23. Misbahuddin M. Rafeeq, and Hussam Aly Sayed Murad, Cystic fibrosis: current therapeutic targets and future approaches, Rafeeq and Murad J Transl Med (2017) 15:84 DOI 10.1186/s12967-017-11939Journal of Translational Medicine.

24. Moss RB. Long-term benefits of inhaled tobramycin in adolescent patients with cystic fibrosis. Chest. 2002; 121:55-63.

25. Konstan MW, Flume PA, Kappler M, et al. Safety, efficacy and convenience of tobramycin inhalation powder in cystic fibrosis patients: the EAGER trial. J Cyst Fibros. 2011; 10:54-61.

26. Flume PA, O'Sullivan BP, Robinson KA, et al. Cystic fibrosis pulmonary guidelines: chronic medications for maintenance of lung health. Am J RespirCrit Care Med. 2007; 176:957-69.

27. Salvatore D, d'Andria M. Effects of salmeterol on arterial oxyhemoglobin saturations in patients with cystic fibrosis. Pediatr Pulmonol.2002; 34:11-5.

28. Robinson M, Regnis JA, Bailey DL, et al. Effect of hypertonic saline,amiloride, and cough on mucociliary clearance in patients with cystic fibrosis. Am J RespirCrit Care Med. 1996; 153:1503-9.
29. Quan JM, Tiddens HA, Sy JP, et al. A two-year randomized, placebocontrolled trial of dornasealfa in young patients with cystic fibrosis with mild lung function abnormalities. J Pediatr. 2001; 139:813-20.

30. McIlwaine MP, Alarie N, Davidson GF, et al. Long-term multicentre randomised controlled study of high frequency chest wall oscillation versus positive expiratory pressure mask in cystic fibrosis. Thorax. 2013; 68:746-51.

31. Colombo C, Ellemunter $\mathrm{H}$, Houwen $\mathrm{R}$, et al. Guidelines for the diagnosis and management of distal intestinal obstruction syndrome in cysticfibrosis patients. J Cyst Fibros. 2011; 10(Suppl 2):S24-8.

32. Stern RC, Eisenberg JD, Wagener JS, et al. A comparison of the efficacy and tolerance of pancrelipase and placebo in the treatment of steatorrhea in cystic fibrosis patients with clinical exocrine pancreatic insufficiency.Am J Gastroenterol. 2000; 95:1932-8.

33. Borowitz D, Robinson KA, et al. Cystic Fibrosis Foundation evidencebasedguidelines for management of infants with cystic fibrosis. J Pediatr.2009; 155:S73-93.

34. Wright CC, Vera YY. Cystic fibrosis. In: DiPiro JT, Talbert RL, Yee GC, et al. eds. Pharmacotherapy: A Pathophysiologic Approach, 10th ed. New York, NY: McGraw-Hill; 2016.

35. Stallings VA, Stark LJ, Robinson KA, et al. Evidence-based practice recommendations for nutrition-related management of children and adults with cystic fibrosis and pancreatic insufficiency: results of a systematic review. J Am Diet Assoc. 2008; 108(5):832-839.

36. Matel, JL. Nutritional management of cystic fibrosis. JPEN. January 2012; 36 (Suppl 1):60S-67S.

37. Schwarzenberg SG, Dorsey J. Pancreatic Enzymes Clinical Care Guidelines. Bethesda, MD: Cystic Fibrosis Foundation; 2013. www.cff.org/Care/Clinical-Care-Guidelines/Nutrition-and-GI-ClinicalCare-Guidelines/Pancreatic-Enzymes-Clinical-Care-Guidelines.2017; 2

38. Ng SM, Jones AP. Drug therapies for reducing gastric acidity in people with cystic fibrosis. Cochrane Database Syst Rev. 2003; 2:CD003424.

39. Francisco MP, Wagner MH, Sherman JM, et al. Ranitidine and omeprazole as adjuvant therapy to pancrelipase to improve fat absorption in patients with cystic fibrosis. J Pediatr Gastroenterol Nutr. 2002; 35:79-83.

40. Farrell PM, White TB, Ren CL, et al. Diagnosis of cystic fibrosis: consensus guidelines from the Cystic Fibrosis Foundation.JPediatr.2017; 181S:S4-S15.

41. Castellani C. Southern KW, Brownlee K, et al. European best practice guidelines for cystic fibrosis neonatal screening. J Cyst Fibros. 2009; 8:153-173.

42. Elborn JS. Cystic fibrosis. Lancet. 2016; 388:2519-2531.

43. Edward De Simone, Jennifer Tilleman, Martin E. Giles, Boussina Moussa. Cystic Fibrosis: Update on Treatment Guidelines and New Recommendations, US Pharm. 2018; 43(5)16-21.

44. Dodge JA, Lewis PA, Stanton M, Wilsher J. Cystic fibrosis mortality and survival in the UK: 1947-2003. Eur Respir J 2007; 29:522-6.

45. Sawyer SM, Phelan PD, Bowes G. Reproductive health in young women with cystic fibrosis: knowledge, behavior and attitudes. J Adolesc Health1995; 17:46-50.

46. Wexler ID, Johannesson M, Edenborough FP, Sufian BS, KeremE. Pregnancy and chronic progressive pulmonary disease. Am J Respir Crit Care Med 2007; 175:300-5.

47. Kent NE, Farquharson DF. Cystic fibrosis in pregnancy. CMAJ 1993; 149:809-13.

48. Hilman BC, Aitken M, Constantinescu M. Pregnancy in patients with cystic fibrosis. ClinObstetGynecol1996; 39:70-86.

49. Alice Panchaud a Ermindo R. Di Paolo b Angela Koutsokerac Ursula Winterfeld d Etienne Weisskopf a David Baud e Alain Sautyc Chantal Csajkaa Safety of Drugs during Pregnancy and Breastfeeding in Cystic Fibrosis Patients.Respiration2016; 91:333-348.

50. Jenny S. Li, Kathy E. Monangai, Alison M. Feathers, Daniel Weiner : The pharmacist's role in supporting people living with cystic fibrosis Journal of the American Pharmacits Association, Published Online: February 04, 2018.

51. Welsh MJ. Cystic fibrosis. In: Schultz SG, ed. Molecular biology of membrane transport disorders. New York: Plenum Press, 1996: 605-23 